\title{
A CLASS OF LINEAR TRANSFORMATIONS WHICH CAN BE WRITTEN AS THE PRODUCT OF PROJECTIONS
}

\author{
JOHN B. HAWKINS AND WILLIAM J. KAMMERER
}

1. Introduction. Over the years there have been many results concerning the structure of linear operators on a complex linear space; most notable among them being the spectral type representations. In this paper we present a factorization type decomposition of certain singular linear operators into the product of a finite number of projections. The easiest stated result in this direction being: An $n \times n$ matrix is singular if and only if it can be written as the product of a finite number of proper projections.

2. Main results. In what follows we shall let $X$ denote a linear space over the real or complex field and by $\nu(T)$ the Hamel dimension of the null space of a linear operator $T$ which maps $X$ into $X$. A projection $P$ of $X$ into $X$ will be said to be a proper projection if and only if $\nu(P) \geqq 1$, i.e. $P$ not the identity operator.

Lemma 1. Let $N: X \rightarrow X$ be a linear operator which is nilpotent of index $q$ and $X_{1}$ a linear subspace of $X$ which has a Hamel basis of the form

$$
\left\{x_{\alpha}, N x_{\alpha}, N^{2} x_{\alpha}, \cdots, N^{q-1} x_{\alpha}: \alpha \in \mathfrak{A}\right\}
$$

where $\mathfrak{A}$ is some indexing set. Then there exists a linear subspace $X_{2}$ which is also invariant under $N$ and for which $X=X_{1} \oplus X_{2}$.

The proof is by induction on the index $q$. When $q=1$ the lemma is trivially true. Assume that the lemma is true for $q-1$. Observe that the restriction $\left.N\right|_{R}$ of $N$ to its range $R$ is nilpotent of index $q-1$ and that the linear subspace $M_{0}=R \cap X_{1}$ is the linear span of vectors of the form

$$
\left\{y_{\alpha}, N y_{\alpha}, \cdots, N^{q-2} y_{\alpha}: y_{\alpha}=N x_{\alpha}, \alpha \in \mathfrak{A}\right\} .
$$

By the induction hypothesis there exists a linear subspace $Y_{0}$ which is invariant under $N$ and $R=M_{0} \oplus Y_{0}$. Letting $Y_{1}=\left\{x: N x \in Y_{0}\right\}$ one writes $N x=y+z$ with $y \in M_{0}$ and $z \in Y_{0}$. Since

$$
y=\sum_{j=1}^{n}\left\{\sum_{i=1}^{q-1} a_{i j} N^{i} x_{\alpha_{j}}\right\}=N\left(\sum_{j=1}^{n} \sum_{i=0}^{q-2} a_{i+1 j} N^{i} x_{\alpha_{j}}\right)=N y_{0} \text { with } y_{0} \in X_{1} \text {, }
$$

Received by the editors March 2, 1967. 
it follows that $N\left(x-y_{0}\right)=z$; that is $\left(x-y_{0}\right) \in Y_{1}$. Thus $x=y_{0}+\left(x-y_{0}\right)$ with $y_{0} \in X_{1}$ and $\left(x-y_{0}\right) \in Y_{1}$ which establishes that $X=X_{1}+Y_{1}$. If $x \in X_{1} \cap Y_{0}$ then $N x \in M_{0}$ and $N x \in Y_{0}$. Thus $N x=0$ and since $x \in X_{1}$,

$$
x=\sum_{j=1}^{m}\left\{\sum_{i=0}^{q-1} b_{i j} N^{i} x_{\alpha_{j}}\right\}
$$

and therefore

$$
0=N x=\sum_{j=1}^{m}\left\{\sum_{i=1}^{q-1} b_{i-1 j} N^{i} x_{\alpha_{j}}\right\} .
$$

From the linear independence of $\left\{N^{i} x_{\alpha_{j}}\right\}$ it follows that $b_{i j}=0$ for $i=0,1, \cdots, q-2, j=1,2, \cdots, m$ so that

$$
x=\sum_{j=1}^{m} b_{q-1 j} N^{q-1} x_{\alpha_{j}}
$$

implying that $x \in M_{0}$. Thus we have proven that $X_{1} \cap Y_{0} \subset M_{0} \cap Y_{0}$, hence $x=0$.

Now recall that $X=X_{1}+Y_{1}, \quad Y_{0} \subset Y_{1}, \quad X_{1} \cap Y_{1} \subset Y_{1}$ and $Y_{0}$ $\cap\left(X_{1} \cap Y_{1}\right)=0$. Let $Z$ be a linear subspace such that $Y_{1}=Z \oplus Y_{0}$ $\oplus\left\{X_{1} \cap Y_{1}\right\}$ and define $X_{2}=Z \oplus Y_{0}$. Observe that $X_{2} \subset Y_{1}, X_{2}$ $\cap\left(X_{1} \cap Y_{1}\right)=0$ implies that $X_{1} \cap X_{2}=0$, showing that $X=X_{1} \oplus X_{2}$. Since $X_{2} \subset Y_{1}, N X_{2} \subset Y_{0} \subset X_{2}$ showing that $X_{2}$ is invariant under $N$.

Lemma 2. If $N: X \rightarrow X$ is a linear mapping which is nilpotent of index $q$ then there exist invariant linear subspaces

$$
Y_{q_{1}}, Y_{q_{2}}, \cdots, Y_{q_{j}}, \quad 0<q_{1}<q_{2}<\cdots<q_{j}=q
$$

of $X$ such that $X=Y_{q_{1}} \oplus Y_{q_{2}} \oplus \cdots \oplus Y_{q_{j}}$, with each subspace $Y_{q_{k}}$ having a Hamel basis of the form

$$
\left\{x_{\alpha}, N x_{\alpha}, \cdots, N^{q_{k}-1} x_{\alpha}: \alpha \in \mathfrak{A}_{k}\right\} \quad(k=1,2, \cdots, j) .
$$

Thus if $E_{k}$ denotes the projection $E x=x_{k}$, if $x=x_{1}+x_{2}+\cdots+x_{j}$ where $x_{k} \in Y_{q_{k}}$, then $N E_{k}$ is nilpotent of index $q_{k}, k=1,2, \cdots, j$.

Proof. Let $\mathfrak{X}$ be the family of all linear subspaces of $X$ which have a Hamel basis of the form $\left\{x_{\alpha}, N x_{\alpha}, \cdots, N^{q_{j}-1} x_{\alpha}\right\}$. Partially ordering this family by inclusion and applying Zorn's lemma establishes the existence of a maximal element $Y_{q_{j}}$ in $\mathfrak{X}$. Lemma 1 guarantees the existence of a linear subspace $M$ which is invariant under $N$ and such that $X=M \oplus Y_{q_{j}}$. Because of the maximality of $Y_{q_{j}}, N$ is nilpotent 
of index $q_{j-1}$ on $M$ with $q_{j-1}<q_{j}$. Repeated application of this argument gives the desired result.

TheOREM 1. If $N: X \rightarrow X$ is a linear nilpotent operator of index $q$ then $N$ can be written as the product of a finite number of projections.

Proof. Let $X=Y_{q_{1}} \oplus Y_{q_{2}} \oplus \cdots \oplus Y_{q_{j}}$ be a decomposition of $X$ of the type discussed in Lemma 2. Relative to a Hamel basis for $Y_{q_{k}}$ of the form $\left\{x_{\alpha}, N x_{\alpha}, \cdots, N^{q_{k}-1} x_{\alpha}: \alpha \in \mathfrak{A}_{k}\right\}$ define $P_{a_{k}}^{(i)}(i=1,2$, $\left.\cdots, q_{k}-1\right)$ to be the projection on

$$
\begin{gathered}
Y_{q_{1}} \oplus \cdots \oplus Y_{q_{k}-1} \oplus \operatorname{span}\left\{x_{\alpha}, \cdots, N^{i-2} x_{\alpha}, N^{i} x_{\alpha}, \cdots, N^{q_{k}-1} x_{\alpha}: \alpha \in \mathfrak{A}_{k}\right\} \\
\oplus Y_{\boldsymbol{q}_{k+1}} \oplus \cdots \oplus Y_{q_{j}}
\end{gathered}
$$

along the span $\left\{N^{i-1} x_{\alpha}-N^{i} x_{\alpha}: \alpha \in \mathfrak{A}_{k}\right\}$. That is, $P_{q_{k}}^{(i)}(i=1,2, \cdots$, $\left.q_{k}-1\right)$ is the linear transformation which transforms $N^{i-1} x_{\alpha}$ into $N^{i} x_{\alpha}$ for each $\alpha \in \mathfrak{A}_{k}$ and the remaining basis elements into themselves. Also define $P_{q_{k}}^{\left(q_{k}\right)}$ to be the projection on

$$
\begin{aligned}
& Y_{q_{1}} \oplus \cdots \oplus Y_{q_{k}-1} \oplus \operatorname{span}\left\{x_{\alpha}, N x_{\alpha}, \cdots, N^{q_{k}-2} x_{\alpha}: \alpha \in \mathfrak{A}_{k}\right\} \\
& \oplus Y_{q_{k+1}} \oplus \cdots \oplus Y_{q_{j}} \text { along }\left\{N^{q_{k}-1} x_{\alpha}: \alpha \in \mathfrak{A}_{k}\right\} .
\end{aligned}
$$

Then $N=\prod_{k=1}^{j}\left\{\prod_{i=1}^{q_{k}} \underset{a_{k}}{P(i)}\right\}$.

Theorem 2. Let $E_{i}: X \rightarrow X, i=1,2$, be two proper projections such that $E_{i} E_{j}=0$ whenever $i \neq j$ and $I=E_{1}+E_{2}$. Let $N: X \rightarrow X$ be a linear nilpotent operator for which $E_{i} N=N E_{i}, i=1,2$, and the linear subspace $E_{2} X$ has a Hamel basis of the form

$$
\left\{y_{\beta}, N y_{\beta}, \cdots, N^{q-1} y_{\beta}: \beta \in \mathfrak{B}\right\} .
$$

If $T=\lambda E_{2}+N, \lambda \neq 0$ and the cardinal of $\mathfrak{B} \leqq \nu(T)$ then $T$ can be written as the product of a finite number of projections.

Proof. The subspaces $X_{1}=E_{1} X$ and $X_{2}=E_{2} X$ are invariant under $T$ and $X=X_{1} \oplus X_{2}$. Since $T E_{1}$ is nilpotent it can be written as the finite product of projections

$$
T E_{1}=\left\{\prod_{i=1}^{m} P_{i}\right\} E_{1}
$$

where $P_{i}, i=1,2, \cdots, m$ is a projection which leaves $X_{2}$ invariant. Let $\left\{x_{\alpha}: \alpha \in \mathfrak{A}\right\}$ be a Hamel basis for the null space of $T$ and extend this linearly independent set to a Hamel basis for $X_{1}$. Since card $\mathfrak{B}$ $\leqq$ card $\mathfrak{A}$, there exists a function $f$ which maps $\mathfrak{B}$ into $\mathfrak{A}$ in a one to one manner. Now define the projections: 
(i) $Q_{1}: x_{f(\beta)} \rightarrow \lambda N^{q-1} y_{\beta}$ for $\beta \in \mathfrak{B}$ and the remaining basis entries remaining invariant,

(ii) $Q_{2}: N^{q-1} y_{\beta} \rightarrow x_{f(\beta)}$ for $\beta \in \mathscr{B}$ and the remaining basis entries remaining invariant.

Also for $k=0,1, \cdots, q-2$ define

(iii) $V_{1}^{(\mathfrak{k})}: x_{f(\beta)} \rightarrow \lambda N^{k} y_{\beta}$ for $\beta \in \mathfrak{B}$ and the remaining basis entries remaining invariant,

(iv) $V_{2}^{(\boldsymbol{k})}: N^{k} y_{\beta} \rightarrow N^{k+1} y_{\beta}$ for $\beta \in \mathfrak{B}$ and the remaining basis entries remaining invariant,

(v) $V_{3}^{(\boldsymbol{k})}: x_{\alpha} \rightarrow 0$ for $\alpha \in \mathfrak{A}$ and $N^{k} y_{\beta} \rightarrow N^{k} y_{\beta}+x_{f(\beta)}$ for $\beta \in \mathfrak{B}$ with the remaining basis entries remaining invariant.

(vi) $P: x_{\alpha} \rightarrow 0$ for $\alpha \in \mathfrak{A}$ and the remaining basis entries remaining invariant.

Then $T=\left\{\prod_{i=1}^{m} P_{i}\right\}\left\{\prod_{k=0}^{q-2} V_{1}^{(\boldsymbol{k})} V_{2}^{(\mathbf{k})} V_{3}^{\mathbf{k}}\right\} Q_{1} Q_{2} P$.

Theorem 3. Let $E_{i}: X \rightarrow X, i=1,2, \cdots, k$ be proper projections such that $I=E_{1}+E_{2}+\cdots+E_{k}$ and $E_{i} E_{j}=0$ whenever $i \neq j$ and let $N: X \rightarrow X$ be a linear nilpotent operator of index $q$ for which $E_{i} N=N E_{i}$ for $i=1,2, \cdots, k$. If

$$
T=\sum_{i=2}^{k} \lambda_{i} E_{i}+N \quad\left(\lambda_{i} \neq 0, i=2,3, \cdots, k\right)
$$

and

$$
\begin{aligned}
-\left\{\nu\left(N^{i} E_{j}\right)-2 \nu\left(N^{i-1} E_{j}\right)+\nu\left(N^{i-2} E_{j}\right)\right\} & \leqq \nu(T), \\
j=2,3, \cdots, k, i & =2,3, \cdots, q+1,
\end{aligned}
$$

then $T$ can be written as the product of a finite number of projections.

Proof. Under either $T$ or $N$ the subspaces $X_{i}=E_{i} X, i=1,2, \cdots$, $k$, are invariant and $X=X_{1} \oplus X_{2} \oplus \cdots \oplus X_{k}$. On $X_{1}, T$ is nilpotent and hence $T E_{1}$ can be written as the finite product of projections

$$
T E_{1}=\left\{\prod_{i=1}^{m} P_{i}\right\} E_{1}
$$

where $P_{i}, i=1,2 . \cdots, m$, are projections which leave $X_{2} \oplus \cdots \oplus X_{k}$ invariant. Lemma 2 implies that there exist subspaces $Y_{p_{1}}, Y_{p_{2}}$, $\cdots, Y_{p_{j}}, 0<p_{1}<\cdots<p_{j} \leqq q$, such that $X_{2}=Y_{p_{1}} \oplus Y_{p_{2}} \oplus \cdots$ $\oplus Y_{p_{j}}$ with each subspace $Y_{p_{i}}$ having a Hamel basis of the form

$$
\left\{x_{\alpha}, N x_{\alpha}, \cdots, N^{p_{i}-1} x_{\alpha}: \alpha \in \mathfrak{A}_{i}\right\}, \quad i=1,2, \cdots, j .
$$

Condition (1) implies that cardinal of $\mathfrak{A}_{i} \leqq \nu(T)$ for $i=1,2, \cdots, j$ and, using a construction similar to that which was used in the proof 
of Theorem 2, there exists for each subspace $Y_{p_{i}}$ a finite product of projections defined on $X$ which equals $T$ on $Y_{p_{i}}$ and leaves the remaining subspaces invariant. The product of these products equals $T$ on $X_{2}$ and leaves the span of $X_{1} \cup X_{3} \cup \ldots \cup X_{k}$ invariant. In a similar manner, there exists a finite product of projections which equals $T$ on $X_{i}(i=3, \cdots, k)$ and leaves the other invariant subspaces unaltered. The product of all the projections described above equals $T$ on $X$.

Let $X$ be a complex Banach space and let $T: X \rightarrow X$ be a continuous linear operator whose resolvent operator $R(\lambda ; T)$ is rational; that is, $R(\lambda, T)=P(\lambda) / Q(\lambda)$ where $P$ is a polynomial with coefficients in $X$ and $q$ is a polynomial with complex coefficients. Denoting the distinct poles of $R(\lambda, T)$ by $\lambda_{1}, \lambda_{2}, \cdots, \lambda_{k}$ with orders $m_{1}, m_{2}, \cdots, m_{k}$ respectively and letting $M_{i}$ be the null space of $\left(T-\lambda_{i} I\right)^{m_{i}}, i=1,2, \cdots, k$ then $T$ is completely reduced by $\left(M_{1}, M_{2}, \cdots, M_{k}\right)$, (see Dunford and Schwartz [1, Chapter 7]). Then letting $E_{i}$ be the projection $E_{i} x=x_{i}, x=x_{1}+x_{2}+\cdots+x_{k}, x_{i} \in M_{i}, i=1,2, \cdots, k$, the operator $T$ can be written as

$$
T=\sum_{i=1}^{k} \lambda_{i} E_{i}+N
$$

where $N$ is the nilpotent operator $N=\sum\left(T-\lambda_{i} I\right) E_{i}$ of index $q=\max \left\{m_{1}, m_{2}, \cdots, m_{k}\right\}$. Thus if $T$ also satisfies condition (1) then according to the previous theorem, $T$ can be written as the finite product of projections. These projections, however, need not be continuous. They will be continuous if and only if their range and null spaces are closed. The following corollary gives an important example when this will always be the case.

Corollary 1. Let $X$ be a complex finite dimensional linear space. Then a linear transformation $T: X \rightarrow X$ is singular if and only if $T$ can be written as the product of a finite number of proper projections.

Proof. Assume that $T$ is a singular linear operator and $X$ is $n$ dimensional. Then there exists a basis $\left\{x_{1}, x_{2}, \cdots, x_{n}\right\}$ for $X$ and a set of integers $0=n_{0}<n_{1}<\cdots<n_{k}=n$ such that $T x_{i}=\lambda_{j} x_{i}+x_{i+1}$ for $n_{j-1}<i<n_{j}$ and $T x_{n_{j}}=\lambda_{j} x_{n_{j}}$, where $\lambda_{j} \in \sigma(T)$ with possible repetition and $\lambda_{1}=0$. The subspaces $X_{j}=$ span of $\left\{x_{i}: n_{j-1}<i \leqq n_{j}\right\}$ are invariant under $T$ and $T=\sum_{i=1}^{k} \lambda_{i} E_{i}+N$ where $N$ is nilpotent satisfying $N x_{i}=x_{i+1}$ for $n_{j-1}<i<n_{j}$ and $N x_{n_{j}}=0$ and $E_{j}$ the projection on $X_{j}$ along $X_{1} \oplus \cdots \oplus X_{j-1} \oplus X_{j+1} \oplus \cdots \oplus X_{k}$. The conclusion follows 
immediately from Theorem 3. Conversely if $T$ is the product of a finite number of proper projections then $T$ is singular.

Corollary 2. Let $X$ be a complex Banach space and let $T: X \rightarrow X$ be a continuous linear operator whose range is a proper finite dimensional subspace of $X$. Then $T$ can be written as the product of a finite number of projections.

Proof. Corollary 1 can be applied if $X$ is finite dimensional. The resolvent of $T$ remains rational if $X$ is not of finite dimension. In fact, the resolvent of $T$ is $R(\lambda, T)=\lambda^{-1}\left\{I+R\left(\lambda, T_{0}\right) T\right\}$ where $R\left(\lambda, T_{0}\right)$ is the resolvent of the restriction $T_{0}$ of $T$ to the range of $T$. If $p(\lambda)$ $=\lambda^{m_{1}}\left(\boldsymbol{\lambda}-\lambda_{2}\right)^{m_{2}} \cdots\left(\lambda-\lambda_{k}\right)^{m_{k}}$ is the minimal polynomial for $T$ then $T$ can be written as $T=\sum_{i=1}^{k} \lambda_{i} E_{i}+N$ where $E_{i}$ and $N$ satisfy the same conditions as those described in (2). $T$ being a bounded linear transformation with a finite dimensional range implies that $T$ is completely continuous. Thus for $i \neq 1$, the null space of $\left(\lambda_{i} I-T\right)^{m_{i}}$ is of finite dimension and condition (1) of Theorem 3 is satisfied.

An important type of operator with a finite dimensional range is an integral operator $T x=\int_{a}^{b} k(s, t) x(t) d t$ with a degenerate kernel $k(s, t)$ $=\sum_{i=1}^{n} \alpha_{i}(s) \beta_{j}(t)$.

3. An application. The iterative scheme

$$
x_{k+1}=\left(I-C_{k} A\right) x_{k}+C_{k} b, \quad k=0,1,2, \cdots,
$$

for solving the linear system $A x=b$, where $A$ is assumed to be an $n \times n$ nonsingular matrix, is called a projective iterative method if and only if each $P_{k}=I-C_{k} A, k=0,1,2, \cdots$, is a proper projection. Many common iterative methods can be viewed as a projective iterative method, such as the point and block Gauss Seidel iterative methods and the method of steepest descent. For further examples see Householder and Bauer [3], Gastinel [2] and Kaczmarz [4]. Corollary 1 allows us to determine which iterative schemes of the form (3) can be written as a projective iterative scheme.

THEOREM 4. The iteration

$$
x_{k+1}=\left(I-C_{k} A\right) x_{k}+C_{k} b, \quad k=0,1,2, \cdots,
$$

can be viewed as a projective iterative scheme if $I-C_{k} A$ is singular for each $k \geqq 0$.

Proof. Since $I-C_{k} A$ is singular, projections $P_{0}, P_{1}, \cdots, P_{m}$ exist such that $I-C_{k} A=\prod_{i=0}^{m} P_{i}$ and $C_{k}=\left\{I-\prod_{i=0}^{m} P_{i}\right\} A^{-1}$. Then the 
next iterate $x_{k+1}$ can be obtained from $x_{k}$ by a finite number of projective type iterations of the form

$$
y_{i+1}=P_{i} y_{i}+\left(I-P_{i}\right) A^{-1} b \text { for } i=0,1, \cdots, m
$$

with $y_{0}=x_{k}$. It is an easy matter to check that $y_{m+1}=x_{k+1}$.

\section{REFERENCES}

1. N. Dunford and J. T. Schwartz, Linear operators, Part I, Interscience, New York, 1958.

2. Nöel Gastinel, Procédé iteratif pour la résolution numérique d'un système d'equations linéaires, C. R. Acad. Sci. Paris 246 (1958), 2571-2574.

3. A. S. Householder and F. L. Bauer, On certain iterative methods for solving linear systems, Numer. Math. 2 (1960), 55-59.

4. S. Kaczmarz, Angenäherte Auflösung von Systemen linear Gleichungen, Bull. Internat. Acad. Polon. Sci. Cl. A (1937), 355-357.

Georgia Institute of Technology 